\title{
Trends in the Incidence and Treatment of Cerebrovascular Diseases in Korea : Part II. Cerebral Infarction, Cerebral Arterial Stenosis, and Moyamoya Disease
}

\author{
Si Un Lee, ${ }^{1,2}$ Tackeun Kim, ${ }^{1,2}$ O-Ki Kwon, ${ }^{1,2}$ Jae Seung Bang, ${ }^{1,2}$ Seung Pil Ban, ${ }^{1,2}$ Hyoung Soo Byoun, ${ }^{1,2}$ Chang Wan O ${ }^{1,2}$ \\ Department of Neurosurgery,' Seoul National University Bundang Hospital, Seongnam, Korea \\ Department of Neurosurgery, ${ }^{2}$ Seoul National University College of Medicine, Seoul, Korea
}

Objective : To analyze trends in the incidence and treatment of diseases associated with ischemic stroke, namely, cerebral infarction (Cl), cerebral arterial stenosis (CASTN), and moyamoya disease (MMD), based on Korean National Health Insurance Service (NHIS) data from 2008 to 2016.

Methods : Data was extracted from the national health-claim database provided by the NHIS for 2008-2016 using International Classification of Diseases codes. The crude and age-standardized incidences of each disease (Cl, CASTN without a history of Cl, and MMD) were calculated; additional analyses were conducted according to age and sex. Trends in the number of patients undergoing treatment according to treatment method were analyzed for each disease using the Korean Classification of Diseases procedure codes.

Results : In 2016, the total number of adults with newly diagnosed Cl was 83939, reflecting a 9.4\% decrease from that in 2008. The agestandardized incidence of $\mathrm{Cl}$ in adults was 153.2 per 100000 person-years in 2016, reflecting a 37.2\% decrease from that in 2008, while that of CASTN was 167.3 per 100000 person-years in 2016, reflecting a $73.3 \%$ increase from that in 2008 . Among treated cases, the number of patients who underwent intra-arterial (IA) treatment, including IA fibrinolysis and mechanical thrombectomy, showed the most prominent increase, increasing at an annual rate of $25.8 \%$. For CASTN, the number of cases treated with carotid artery stenting or balloon angioplasty (CAS) showed the most prominent increase, increasing at a rate of $69.8 \%$ over the 9 -year period. For MMD, the total number of patients with newly diagnosed MMD and that with adult MMD demonstrated significantly increasing trends, while the number of pediatric patients with newly diagnosed MMD declined by $18.0 \%$ over the 9 -year period. The age-standardized incidences of pediatric and adult MMD in 2016 were 2.4 and 3.4 per 100000 person-years, respectively.

Conclusion : Although the incidence of $\mathrm{Cl}$ showed a declining trend over a 9-year period, the number and proportion of patients treated for $\mathrm{Cl}$ increased. Meanwhile, the incidence of CASTN and the number of patients treated for CASTN have demonstrated increasing trends since 2008. On the other hand, the number of patients diagnosed with pediatric MMD decreased, despite no significant change in the incidence. In contrast, the number of patients and the incidence of adult MMD increased. These trends reflect changes in the population structure, gains in the accessibility of imaging examinations, and the development of endovascular techniques.

Key Words : Incidence $\cdot$ National health service $\cdot$ Cerebral infarction $\cdot$ Caritid stenosis · Moyamoya disease.

- Received : September 12, 2018 •Accepted : November 26, 2018

- Address for reprints : Chang Wan Oh

Department of Neurosurgery, Seoul National University Bundang Hospital, 82 Gumi-ro 173beon-gil, Bundang-gu, Seongnam 13620, Korea

Tel : +82-31-787-7169, Fax : +82-31-787-4059, E-mail : wanogh@snubh.org, ORCID : https://orcid.org/0000-0001-7311-7072

This is an Open Access article distributed under the terms of the Creative Commons Attribution Non-Commercial License (http://creativecommons.org/licenses/by-nc/4.0) which permits unrestricted non-commercial use, distribution, and reproduction in any medium, provided the original work is properly cited. 


\section{INTRODUCTION}

Stroke, the third most common cause of death worldwide, is rapidly emerging as a global health problem, and will be of even greater importance in the future due to ongoing demographic changes, such as the ageing of the population ${ }^{14,24,38)}$. Additionally, the greatest burden of stroke continues to reside in developing countries, despite decreased stroke-related mortality rates in developed countries ${ }^{10,35)}$. Ischemic stroke (IS) accounts for a large proportion $(76.4 \%$ to $64.9 \%)$ of all stroke cases $^{4,18,20,23,24)}$, and underlies an increasing trend in the absolute number of disability-adjusted life-years since $1990^{10)}$. Cerebral arterial stenosis (CASTN) and moyamoya disease (MMD) are important causes of IS, especially in Asian populations ${ }^{3,6,11,22,33,40)}$. Moreover, the risk of stroke in patients with CASTN remains high, even with medical therapy using antiplatelets or anticoagulants ${ }^{7}$.

Although nationwide studies on the epidemiology of IS $^{4,18,20,23,24,26)}$ and $\mathrm{MMD}^{3,6,22,25,28,33)}$ have been reported, there are no recent nationwide studies on CASTN. Furthermore, no large-scale study has analyzed trends in the associated treatment methods. The National Health Insurance Service (NHIS) in Korea is a nationwide insurance system covering $97 \%$ of the Korean population. NHIS data are based on insurance claims sent from healthcare institutions; details of the information stored in the NHIS database have been previously described $^{29,34}$. As Korean NHIS data covers a single-ethnicity population of 50 million people, it represents the largest population in epidemiologic studies. Furthermore, the International Classification of Diseases (ICD)-10-based definitions in Korea for cerebrovascular diseases (CVD) have been validated in a previous study ${ }^{36}$. Thus, Korean NHIS data provide an advantage in studying the epidemiology of CVD.

Therefore, the present study utilized Korean NHIS data to analyze trends in the incidence and treatment of CVD associated with IS (namely, cerebral infarction [CI], CASTN, and MMD) from 2008 to 2016 in Korea.

\section{MATERIALS AND METHODS}

\section{Data extraction}

Data were extracted from the national health-claim database provided by the NHIS for 2005-2016. We selected all pa- tients newly diagnosed with CI, CASTN, or MMD (ICD-10 codes of I63, I65 or I66, and V128, respectively). Patients diagnosed with CI, CASTN, or MMD during the preceding 3 years at any clinic or hospital were excluded to reduce prevalent cases. Therefore, 2008 was used as the index year, and thus, the total observation period of the reconstructed cohort was from January 2008 to December 2016. Furthermore, we restricted the data extraction to tertiary referral general hospitals (TRGHs), general hospitals (GHs), semi-hospitals, and primary medical institutions, as such institutions can obtain a precise diagnosis. Other medical institutions where medical information was not clear were excluded. This study was approved by Institutional Review Board at Seoul National University Bundang Hospital (X-1703-388-909), which waived informed consent.

Additionally, to avoid overestimation of the population and to include only newly incident cases, data extraction was restricted to patients who underwent specific diagnostic imaging modalities, namely, computed tomography angiography (CTA), magnetic resonance angiography (MRA), or digital subtraction angiography, within 4 weeks from the initial diagnosis.

\section{Study populations according to disease}

\section{$\mathrm{Cl}$}

Patients newly diagnosed with CI were identified by an ICD code of I63. Only patients with $\geq 3$ days of hospitalization or death within 3 days of hospitalization with an ICD-10 code of I63 were included in an effort to exclude patients with old CI. Furthermore, we only included patients who underwent diagnostic imaging, such as CT with a contrast agent, brain CTA, or brain magnetic resonance imaging (MRI) with or without MRA, within 7 days from admission to restrict the analysis to patients diagnosed with acute or subacute CI, rather than old $\mathrm{CI}^{5,16}$. Furthermore, we excluded patients with a history of CI or other CVD within 3 years prior to the index period, and patients with other causes of brain injury able to provoke the CI (e.g., brain trauma and brain tumor). Additionally, we excluded pediatric patients under 20 years of age, as the pathophysiology of pediatric CI is different from that of adult $\mathrm{CI}^{12,15,32,43)}$.

\section{Cerebral artery stenosis}

Patients diagnosed with CASTN were identified by ICD 
codes I65 and I66, including both extracranial and intracranial artery stenosis. Patients with an ICD code of I63 were excluded to restrict the analysis to only those without a history of CI. Furthermore, we excluded patients with a history of cerebral arterial dissection (ICD code : I720, I725, I726, I670, or I671), primary or secondary vasculitis (ICD code : I68, L93, L95, M05, M30-32, M35, or D57), cerebral autosomal dominant arteriopathy with subcortical infarcts and leukoencephalopathy (CADASIL) (ICD code : I673), sickle cell disorder (ICD code : D57), or MMD (ICD code : I675 and V128) to restrict the analysis to only those with atherosclerotic stenosis or occlusion $^{13}$. Furthermore, we excluded patients with a history of CASTN or other CVD within 3 years prior to the index period, and patients with brain trauma or brain tumors. Additionally, we excluded pediatric patients under 20 years of age as the etiology differs from that in adult patients.

\section{Moyamoya disease}

Patients diagnosed with MMD were identified by an ICD code of I67.5 with V128. Patients with a history of MMD within 3 years prior to the index period were excluded.

\section{Data analysis}

\section{Incidence of cerebrovascular diseases}

Data manipulation and extraction were conducted in SAS version 9.4 (SAS Institute Ins., Cary, NC, USA). The number of CVD cases diagnosed for the first time was calculated for each year from 2008 to 2016, and the annual crude incidence was calculated using the total population of each year, corrected for the number of deaths and births in each year. The annual crude incidences of CI, CASTN, and MMD were directly calculated based on the cohort's observation data using the following formula : annual crude incidence $=$ number of events/total observation period $\times 100000$. The age-standardized incidence was obtained by applying the proportions of sex and age groups from the census data of 2008 in Korea to adjust for changes in the population structure, using the following formula : $\sum_{i} P_{i} \times I_{i}\left(\mathrm{P}_{\mathrm{i}}=\right.$ proportion of each age / sex group in the national population; $\mathrm{I}_{\mathrm{i}}=$ incidence of each age / sex group in the cohort population). Additionally, we calculated age-specific incidences to obtain the crude incidence for each age group, with age-groups defined in 10-year periods.

\section{Treatment for cerebrovascular diseases}

Trends in the number of patients undergoing treatment according to the treatment method were analyzed for each of the CVD. Changes in the number of patients undergoing treatment by region and hospital size were also analyzed. Treatments for CI or CASTN were identified by the following procedure codes in the Korean Classification of Diseases (KCD) : intra-arterial (IA) treatment, including IA fibrinolysis and mechanical thrombectomy, M6631-M6633; intracranial arterial stenting and balloon angioplasty (ICAS), M6594 and M6602; carotid artery stenting and balloon angioplasty (CAS), M6594 and M6602; decompressive craniectomy, N0333; direct bypass (DB), S4661; carotid endarterectomy (CEA), O0226, O0227, and O2066. Treatments for MMD included DB (KCD code : S4661) and indirect bypass (IB; KCD code : S4662).

\section{Statistical analysis}

Statistical analyses were performed using SPSS version 22 (IBM Corp., Armonk, NY, USA). Spearman's rank-order correlation was used to evaluate the linear trend; $p$-values and Spearman's coefficient (r) are reported. The Wilcoxon signedrank test was used to confirm changes in the variables for each year. A $p$-value $<0.05$ was regarded as statistically significant.

\section{RESULTS}

\section{Cl}

\section{Incidence of $\mathrm{Cl}$}

A total of 92691 and 83939 adults were newly diagnosed with CI in 2008 and 2016, respectively, reflecting a significant decreasing trend over the 9-year period $(p<0.01, \mathrm{r}=-0.967)$. The crude and age-standardized incidences in 2016 were calculated as 199.6 per 100000 person-years and 153.2 per 100000 person-years, respectively, both reflecting decreasing trends from 244.2 per 100000 person-years in $2008(p<0.01$, $\mathrm{r}=-0.983$ ) (Fig. 1A).

The number of patients diagnosed with CI in their 80 s increased by $22.5 \%$ over the 9-year period, but declined for other age groups. However, the age-specific incidences showed decreasing trends over the 9-year period for all age groups, and the age-specific incidence for patients older than 60 years exceeded the overall crude incidence. The proportion of pa- 
tients aged over 60 years among those diagnosed with CI was $80.6 \%$ in 2016, reflecting a significant increase from that in $2008(p<0.01, \mathrm{r}=0.883)$ (Fig. $1 \mathrm{~B}$ and C).

Additionally, the crude incidence was significantly higher in men than in women ( $p=0.018$ ). In 2016, the crude incidence of $\mathrm{CI}$ in men exceeded that in women among patients in their 30 s or older, and was 2.5 times that in women among patients in their 50s (Fig. 1B and C).

\section{Treatments for $\mathrm{Cl}$}

The number of adult patients treated for CI in the acute or subacute phase increased annually over the 9-year period, increasing at an average annual rate of $15.5 \%(p<0.01, \mathrm{r}=1.0)$. Accordingly, the ratio of patients treated for CI in the acute or subacute phase increased from $3.4 \%$ in 2008 to $8.4 \%$ in 2016 , which reflected the increased ratio of patients treated with endovascular or surgical intervention $(p<0.01, r=1.0)$. The number of patients who underwent DB increased steadily until
2014 ( $p=0.016, \mathrm{r}=0.847$ ) and then showed a decreasing trend, with only 146 patients $(0.2 \%$ of the patients with $\mathrm{CI})$ treated by DB in 2016. The number of patients who underwent CEA increased steadily until $2011(p=0.20, \mathrm{r}=0.800)$ and then plateaued, with only 141 patients $(0.2 \%)$ treated with CEA in 2016. The number of ICAS cases has decreased since 2013 $(p<0.01, \mathrm{r}=-1.0)$, with only $496(0.6 \%)$ ICAS cases in 2016 . The number of CAS cases increased 2.4-fold over a period of 5 years (2008 to 2013) $(p<0.01, r=0.833)$ and then plateaued (Fig. 2).

Notably, the number of patients who underwent IA treatment (including IA fibrinolysis and mechanical thrombectomy) showed a prominent increase (average annual rate, 25.8\%), and 4282 patients with CI underwent IA treatment in $2016(p<0.01, r=1.0)$. The number of elderly patients aged over 60 years who underwent IA treatment increased over the 9 -year period, increasing at an average annual rate of $28.0 \%$ $(p<0.01, r=1.0)$, and comprised $77.1 \%$ of all patients who un-

(A)
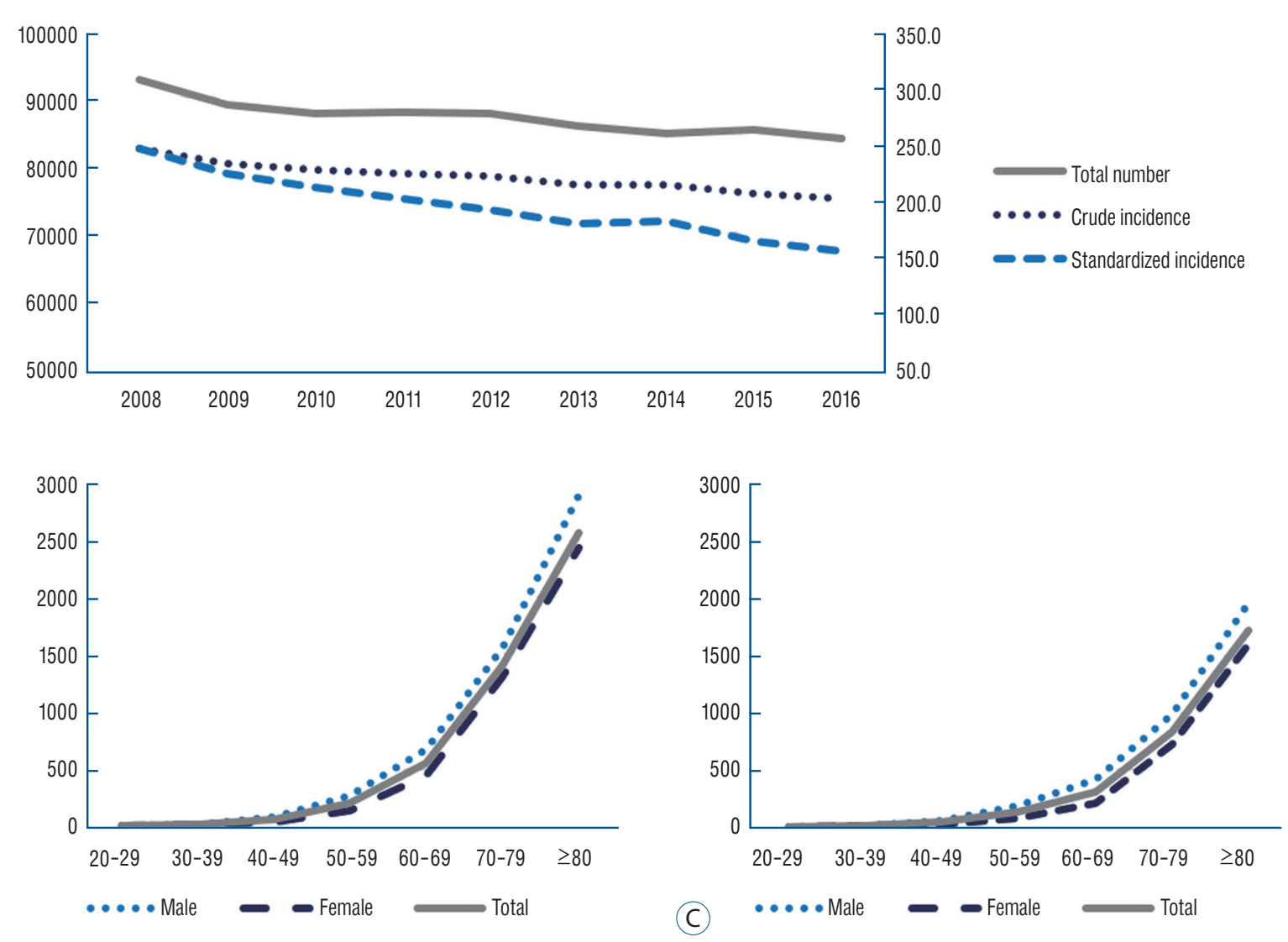

Fig. 1. Incidence of cerebral infarction (Cl). A : Total number of patients diagnosed with $\mathrm{Cl}$, the crude incidence, and age-standardized incidence show decreasing trends over the 9 -year observation period. B and C : The age-specific incidences show decreasing trends over the 9 -year observation period for all age groups. The crude incidence was significantly higher in men than in women. 


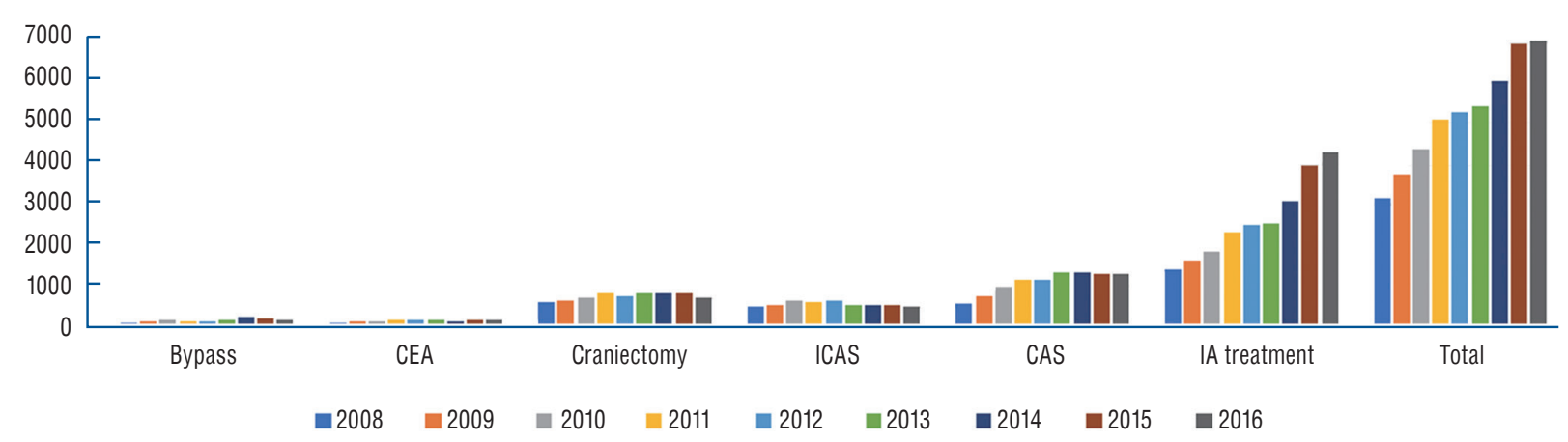

Fig. 2. Treatments for $\mathrm{Cl}$. Total number of adult patients treated for acute or subacute $\mathrm{Cl}$ increased annually, at an annual rate of $15.5 \%$. The number of patients who underwent DB increased steadily until 2014 and then showed a decreasing trend. The number of patients who underwent CEA increased steadily until 2011 and then plateaued. The number of ICAS cases has decreased since 2013. The number of CAS cases increased 2.4-fold over a period of 5 years (until 2013) and then plateaued. The number of patients who underwent IA treatment, including IA fibrinolysis and mechanical thrombectomy, has increased at an annual rate of $25.8 \%$ over 9 years. CEA : carotid endarterectomy, ICAS : intracranial arterial stenting and balloon angioplasty, CAS : carotid artery stenting and balloon angioplasty, IA : intra-arterial, $\mathrm{Cl}$ : cerebral infarction, DB : direct bypass.

(A)
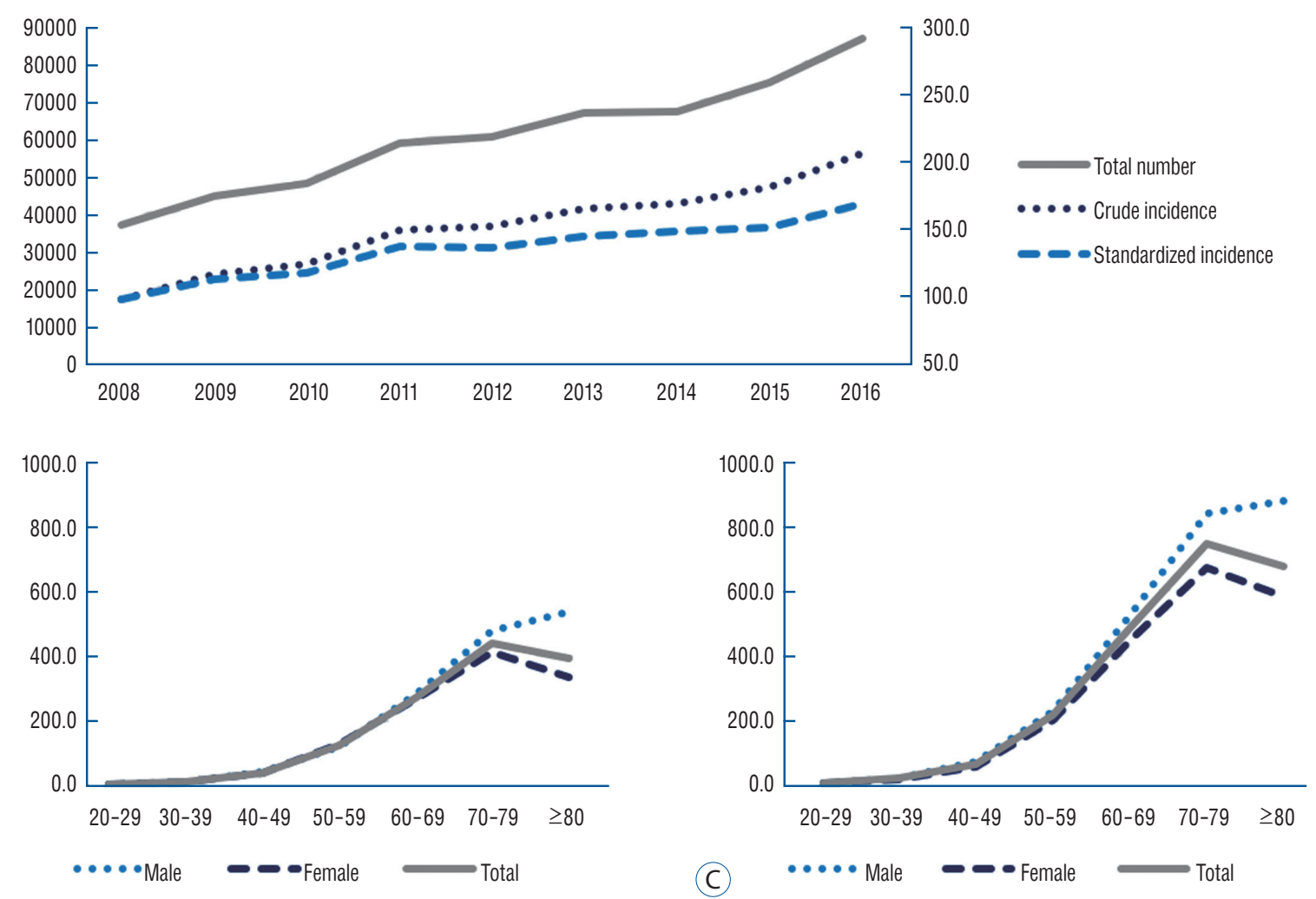

Fig. 3. Incidence of cerebral arterial stenosis (CASTN). A : Total number of patients diagnosed with CASTN, the crude incidence, and age-standardized incidence show significantly increasing trends over a 9-year period. B and C : The age-specific incidence of CASTN increased annually for all age groups, and was the highest for patients in their 70s. The crude incidence of CASTN in men exceeded that in women among patients in their 30 s or older, and was 1.5 times more than that in women among patients in their $80 \mathrm{~s}$. 
derwent IA treatment for CI in 2016 (Fig. 2).

\section{Cerebral artery stenosis}

\section{Incidence of cerebral artery stenosis}

A total of 36644 and 86178 adults were newly diagnosed with CASTN in 2008 and 2016, respectively, reflecting at an average annual increasing rate of $16.9 \%(p<0.01, \mathrm{r}=1.0)$. Both the crude incidence and age-standardized incidence increased steadily, and were calculated as 204.9 per 100000 person-years and 167.3 per 100000 person-years, respectively, in 2016 $(p<0.01, r=0.983)$ (Fig. 3A). Overall, the incidence of CASTN increased annually for all age groups. The age-specific incidence was the highest for those in their 70s, and the age-specific incidence in patients aged over 50 years exceeded the overall crude incidence. The proportion of patients aged over 60 years among those diagnosed with CASTN was $68.9 \%$ in 2016 , reflecting a increase from $65.6 \%$ in $2008(p=0.05$, $\mathrm{r}=0.667$ ). Additionally, the crude incidence of CASTN in men exceeded that in women among patients in their 30s or older, and was 1.5 times that in women among patients in their $80 \mathrm{~s}$ $(p=0.028)$ (Fig. 3B and C).

\section{Treatments for cerebral artery stenosis}

The number of adult patients treated for CASTN increased annually over the 9-year period, increasing at an average annual rate of $5.2 \%(p<0.01, \mathrm{r}=0.917)$. Nevertheless, the ratio of patients treated for CASTN decreased from $4.1 \%$ in 2008 to $2.5 \%$ in $2016(p<0.01, r=-0.983)$, as the increase in the inci- dence of CASTN was superior to the increase in the number of patients treated. The number of patients who underwent DB increased steadily until $2013(p=0.072, r=0.771)$ and then showed a decreasing trend $(p<0.01, \mathrm{r}=-1.0)$, with only $145 \mathrm{pa}-$ tients $(0.1 \%$ of patients with CASTN) treated by DB in 2016. The number of patients who underwent CEA increased steadily until $2012(p=0.01, \mathrm{r}=1.0)$ and then plateaued, with only 458 patients $(0.5 \%)$ treated by CEA in 2016 . The number of ICAS cases has decreased since 2012 ( $p=0.329, \mathrm{r}=-0.486$ ), with 200 cases $(0.2 \%)$ in 2016, while the number of CAS cases increased 1.7-fold over the 9-year period ( $p<0.01, \mathrm{r}=0.983)$, with 1328 cases (1.5\%) in 2016 (Fig. 4).

\section{Moyamoya disease}

\section{Incidence of moyamoya disease}

A total of 1101 and 1679 patients were diagnosed with MMD in 2008 and 2016, respectively, reflecting an annual increasing rate of $6.6 \%$ over the 9 -year period $(p<0.01, \mathrm{r}=0.983)$. The crude and age-standardized incidences in 2016 were calculated as 3.2 per 100000 person-years and 3.1 per 100000 person-years, respectively, both reflecting increasing trends from 2.2 per 100000 personyears in 2008 ( $p<0.01, \mathrm{r}=0.983$ ) (Fig. $5 \mathrm{~A})$.

The number of pediatric patients with newly diagnosed MMD declined by $18.0 \%$ over the 9-year period, with 243 pediatric patients diagnosed with MMD in $2016(p<0.01$, $\mathrm{r}=-0.912$ ). For pediatric MMD, the crude and age-standardized incidences showed no significant change from 2008 to 2016, and both were calculated as 2.4 per 100000 person-years

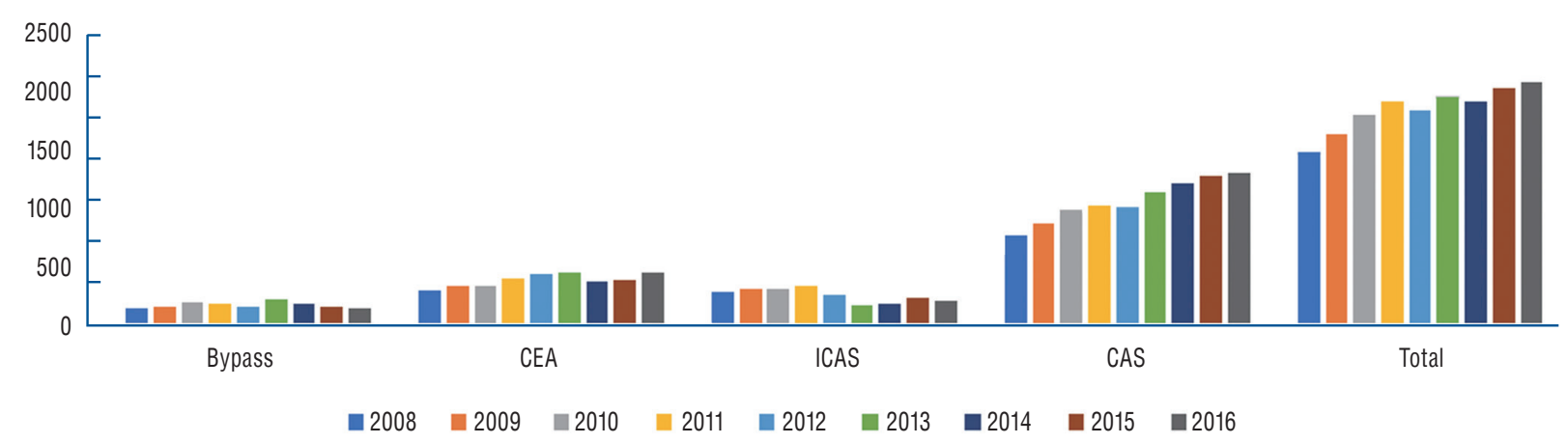

Fig. 4. Treatments for CASTN. Total number of adult patients treated for CASTN increased annually, at an annual rate of $5.2 \%$. The number of patients who underwent DB increased steadily until 2013 and then showed a decreasing trend. The number of patients who underwent CEA increased steadily until 2012 and then plateaued. The number of ICAS cases has decreased since 2012, while the number of CAS cases has increased by 1.7-fold over a 9-year period. CAS : carotid artery stenting and balloon angioplasty, ICAS : intracranial arterial stenting and balloon angioplasty, CAS : carotid artery stenting and balloon angioplasty, CASTN : cerebral artery stenosis, DB : direct bypass. 
in 2016 ( $p=0.70, \mathrm{r}=0.150)$. In contrast, the number of adult patients with newly diagnosed MMD increased by $78.4 \%$ over the 9-year period ( $p<0.01, \mathrm{r}=0.950$ ), with 1,436 adult patients diagnosed with MMD in 2016. For adult MMD, the crude and age-standardized incidences showed significantly increasing trends from 2008 to 2016 ( $p<0.01, \mathrm{r}=0.983)$, and both were calculated as 3.4 per 100000 person-years in 2016 (Fig. 5A). The age-specific incidence was the highest for patients in their 50 s. The proportion of patients aged over 60 years among those diagnosed with MMD increased 2.0-fold over 9 years $(p<0.01, \mathrm{r}=0.917)$ and accounted for $16.7 \%$ of the patients with MMD in 2016. In general, a bimodal shape was observed, peaking in the teens and 50s, but this pattern was more pronounced in women than in men. In 2016, the crude incidence was 2.3 per 100000 person-years and 4.1 per 100000 personyears in men and women, respectively, and was 2.1 times higher in women than in men among patients in their $50 \mathrm{~s}$ ( $p=0.018)$ (Fig. 5B and C).

\section{Treatments for moyamoya disease}

Operations for pediatric MMD declined significantly over the 9-year period ( $p<0.01, \mathrm{r}=-0.820$ ), with 399 and 313 operations performed in 2008 and 2016, respectively. For pediatric MMD, the number of DB cases, including cases of combined bypass (CB), increased steadily over the 9-year period, with 40 cases of DB performed in 2016. Meanwhile, the total number of revascularization surgeries for adult MMD increased to 389 by $2012(p<0.01, r=0.964)$ and then decreased to 364 in 2016. The number of DB cases, including CB cases, peaked at 266 in $2014(p=0.188, \mathrm{r}=0.700)$ and then decreased to 250 in 2016 $(p=0.01, \mathrm{r}=-1.0)$. The proportion of cases in which IB was performed alone decreased from $62.5 \%$ in 2008 to $31.3 \%$ in 2016 $(p<0.01, \mathrm{r}=-0.850)$.

(A)
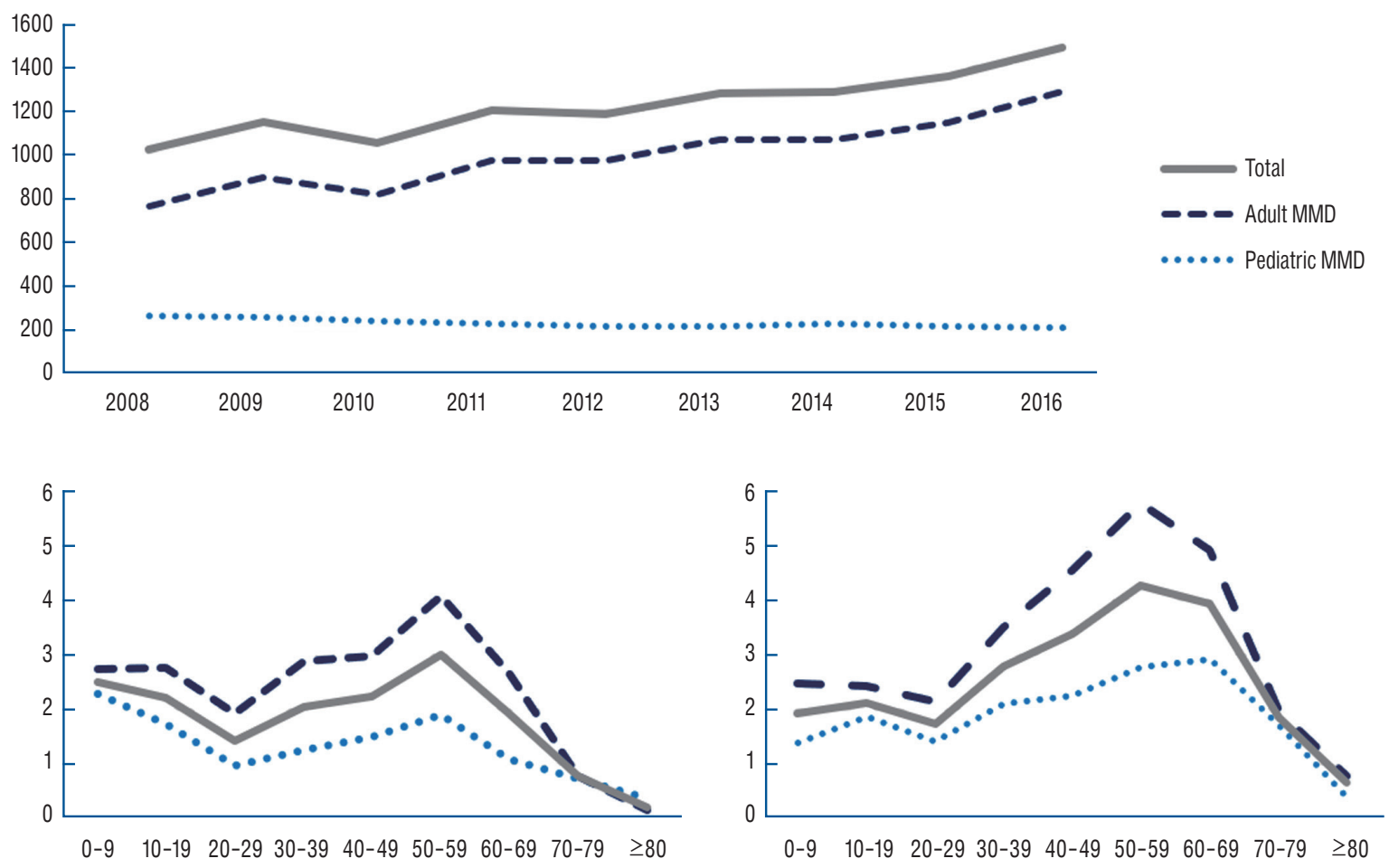

(B)

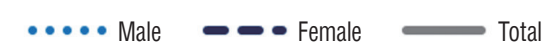

(C)

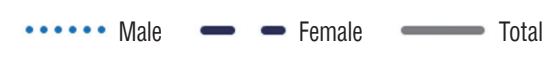

Fig. 5. Incidence of moyamoya disease (MMD). A : The total number of patients with newly diagnosed MMD and that with adult MMD demonstrated increasing trends, while the number of pediatric patients with newly diagnosed MMD declined by $18.0 \%$ over a 9 -year period. B and C : While the agespecific incidences in pediatric patients with MMD decreased, that in adult patients increased over a 9-year period. A bimodal shape is observed, peaking in the teens and 50s, but this pattern is more pronounced in women than in men. 


\section{DISCUSSION}

\section{$\mathrm{Cl}$ and cerebral artery stenosis}

A previous study utilizing Korean NHIS data from 2005 to 2010 showed that the incidence of IS increased to 95210 in 2007 and then declined ${ }^{18)}$. According to the present study, the number of patients diagnosed with IS was 92872 in 2008 and 84084 in 2016, decreasing at an average rate of $1.2 \%$ per year. In addition, the decrease in the age-standardized incidence was larger than that for the crude incidence, and in 2016, the age-standardized incidence was 117.0 per 100000 personyears. Thus, the actual incidence of $\mathrm{CI}$ is decreasing, despite compensation for changes in the population structure due to aging. Similar to these trends in Korea, substantial decreases in stroke incidence have been reported in multicenter cohort studies conducted in the United States ${ }^{23)}$. Decreasing trends have also been reported in Europe, New Zealand, Australia, and Japan ${ }^{4,17,20,39,42)}$. The more successful control of risk factors in the last few decades (mainly hypertension control starting in the 1970s, and later, hypertension treatment combined with smoking cessation, control of diabetes and dyslipidemia, and treatment of atrial fibrillation) may have resulted in lower stroke incidence and less severe strokes ${ }^{26}$. The results of the present study also showed that the diagnosis and treatment of CASTN increased 2.4 times and 1.4 times, respectively, over the 9-year observation period. This increase in diagnosis and preventative treatment may have helped control the risk factors of CI, further lowering the incidence of CI.

The number of patients who underwent treatment for CI increased by an average of $18.3 \%$ per year for 9 years, which was largely due to increases in carotid angioplasty and IA treatment. In particular, the number of patients undergoing IA treatment has been on the rise since 2013, which coincides with changes in the guideline for acute ischemic stroke (AIS) ${ }^{211}$. In the 2013 AIS guideline, the effectiveness of mechanical thrombectomy was established, which differed from that in previous guidelines ${ }^{2,21}$. The guideline was further revised in 2018, and mechanical thrombectomy was shown to be reasonable for select patients, even within 24 hours of the last known normal; thus, the indication of mechanical thrombectomy is wider and the number of patients undergoing IA treatment is expected to increase further ${ }^{37}$. As for ICAS, the SARIS study reported a favorable outcome for intracranial stenosis in $\mathrm{AIS}^{30,31)}$, but no other evidence, such as that from a randomized clinical trial, was obtained. Furthermore, the
SAMMPRIS study reported that medical treatments were superior for symptomatic intracranial stenosis ${ }^{8,99}$. These reports explain why the number of patients undergoing ICAS for CI and CASTN is decreasing. Furthermore, several studies have reported CAS as effective for symptomatic carotid stenosis ${ }^{1}$. Additionally, the number of studies reporting the effectiveness and safety of CAS for AIS are growing ${ }^{19,27,41,44)}$. However, the evidence supporting CAS for AIS was not established in the 2018 guideline. Despite this, CAS accounted for $20.7 \%$ of all CI treatments in 2016, reflecting a 2.3-fold increase from 2008.

An increase in the number of available hospitals is considered to be one of the major causes of the increased number of CAS and IA treatments (e.g., IA fibrinolysis, mechanical thrombectomy). Over the 9-year observation period, the number of GHs able to perform IA treatment increased 2.0fold, while the number of patients who underwent IA treatment in a GH increased 3.7-fold. Meanwhile, the number of patients who underwent IA treatment in a TRGH increased 2.8 -fold, even though there was no significant change in the number of TRGHs able to perform IA treatment. The number of available TRGHs and GHs were 40 and 67, respectively, in 2016. In contrast, the number of available TRGHs and GHs were 42 and 90 in 2016, reflecting increases of 7.7\% and 95.7\%, respectively. Interestingly, the number of CAS performed in GHs has exceeded that in TRGHs since 2013.

\section{Moyamoya disease}

In the current study, the crude and age-standardized incidences for MMD in 2016 were calculated as 3.2 per 100000 person-years and 3.1 per 100000 person-years, respectively, and the number of patients newly diagnosed with MMD increased by $52.5 \%$ since 2008 . Although both the crude and age-standardized incidences of pediatric MMD in 2016 (2.4 per 100000 person-year) were not significantly different from those in 2008, those for adult MMD significantly increased from 2.1 per 100000 person-years in 2008 to 3.4 per 100000 person-years in 2016. According to data from the Korean NHIS, the number of health insurance subscribers under the age of 20 years decreased $15.5 \%$ from 11920343 in 2008 to 10070627 in 2016. This explains why there was no significant change in the crude incidence despite an $18.0 \%$ decrease in the number of newly diagnosed pediatric MMD over the 9 -year period. While the number of diagnoses for adult MMD increased, the number of surgeries has been decreasing since 
2012. This is assumed to be due to an increased detection rate in asymptomatic MMD, as a result of increased health screening. The incidence trend in the present study is similar to that in a previous Korean nationwide study conducted from 2006 to 2011, and the incidence of MMD has increased steadily since $2011^{3}$. Based on these results, it is deduced that the incidence, as reported previously and in the present study, is close to the detection rate, and that the detection rate increases as the accessibility to imaging modalities increases. Although a previous Japanese study reported an incidence of 0.54 per 100000 person-years in 2003, which is lower than the rate found in the present study, the Japanese study analyzed data from a survey conducted in hospitals in 2003, with a response rate of $56.8 \%{ }^{25}$. Additionally, a nationwide population-based study in Taiwan conducted from 2000 to 2011 reported an annual incidence of 0.15 per 100000 person-years, with increasing incidence in adults but not in children ${ }^{6}$. In a multicenter study in China, an increasing incidence rate of MMD was observed during the period of 2000-2007, with an average detection rate of 0.43 per 100000 persons-years ${ }^{33}$. Although the incidence of MMD in the present study was higher than that in other nationwide studies, the present study is based on Korean NHIS data, which represent $97 \%$ of the nation's population, and the results reflect the most recent trends. Therefore, we believe the present results are reliable and meaningful.

\section{Limitations}

The present study has several limitations, mainly related to the use of claim data rather than medical records. First, in patients with a diagnosis of $\mathrm{CI}$, there is a limit to clearly knowing the onset time. To overcome this limitation, we only included patients with $\geq 3$ days of hospitalization or death within 3 days of hospitalization with the appropriate ICD-10 code to exclude patients with old infarctions. Furthermore, we included only patients who underwent diagnostic imaging, such as CT with a contrast agent, brain CTA, or brain MRI, with or without MRA within 7 days from admission ${ }^{5,16)}$. Second, among patients diagnosed with CASTN, it is possible that patients with pathologies other than atherosclerosis were included, as the claim data does not include imaging data. However, we excluded patients with other etiologies, such as dissection, vasculitis, CADASIL, sickle-cell anemia, or MMD. Furthermore; as the incidence of other etiologies is very $\operatorname{low}^{13}$, their impact on the results is not expected to be significant.

\section{CONCLUSION}

Although the incidence of CI demonstrated a declining trend over the 9-year observation period, the number of patients treated for CI increased 2.2-fold. The incidence of CASTN and the number of patients treated for CASTN both increased over the 9-year period. On the other hand, the number of patients diagnosed with pediatric MMD decreased, despite no significant change in the incidence. In contrast, the number of patients diagnosed with adult MMD and its incidence have increased over the 9-year period. These trends reflect changes in the population structure, increases in the accessibility of imaging examinations, and the development of endovascular techniques. An understanding of these trends may aid in the prevention of stroke and the establishment of related healthcare policies.

\section{CONFLICTS OF INTEREST}

No potential conflict of interest relevant to this article was reported.

\section{INFORMED CONSENT}

This type of study does not require informed consent.

\section{AUTHOR CONTRIBUTIONS}

\author{
Conceptualization : CWO \\ Data curation : SUL, TK \\ Formal analysis : SUL \\ Funding acquisition : $\mathrm{CWO}$ \\ Methodology : SUL \\ Project administration : JSB, OKK \\ Visualization: SPB, HSB \\ Writing - original draft : SUL \\ Writing - review \& editing : SUL, CWO
}

\section{- Acknowledgements}

This study was supported by the Policy Research Funds of 
the Korean Neurosurgical Society.

\section{References}

1. Abbott AL, Paraskevas KI, Kakkos SK, Golledge J, Eckstein HH, DiazSandoval LJ, et al. : Systematic review of guidelines for the management of asymptomatic and symptomatic carotid stenosis. Stroke 46 : 32883301, 2015

2. Adams HP Jr, del Zoppo G, Alberts MJ, Bhatt DL, Brass L, Furlan A, et al. : Guidelines for the early management of adults with ischemic stroke: a guideline from the American Heart Association/American Stroke Association Stroke Council, Clinical Cardiology Council, Cardiovascular Radiology and Intervention Council, and the Atherosclerotic Peripheral Vascular Disease and Quality of Care Outcomes in Research Interdisciplinary Working Groups: the American Academy of Neurology affirms the value of this guideline as an educational tool for neurologists. Stroke 38 : 1655-1711, 2007

3. Ahn IM, Park DH, Hann HJ, Kim KH, Kim HJ, Ahn HS : Incidence, prevalence, and survival of moyamoya disease in Korea: a nationwide, population-based study. Stroke 45 : 1090-1095, 2014

4. Anderson CS, Carter KN, Hackett ML, Feigin V, Barber PA, Broad JB, et al. : Trends in stroke incidence in Auckland, New Zealand, during 1981 to 2003. Stroke $36: 2087-2093,2005$

5. Campbell BC, Purushotham A, Christensen S, Desmond PM, Nagakane $Y$, Parsons MW, et al. : The infarct core is well represented by the acute diffusion lesion: sustained reversal is infrequent. J Cereb Blood Flow Metab 32 : 50-56, 2012

6. Chen PC, Yang SH, Chien KL, Tsai IJ, Kuo MF : Epidemiology of moyamoya disease in Taiwan: a nationwide population-based study. Stroke 45 : 1258-1263, 2014

7. Chimowitz MI, Kokkinos J, Strong J, Brown MB, Levine SR, Silliman S, et al. : The warfarin-aspirin symptomatic intracranial disease study. Neurology 45 : 1488-1493, 1995

8. Chimowitz MI, Lynn MJ, Derdeyn CP, Turan TN, Fiorella D, Lane BF, et al. : Stenting versus aggressive medical therapy for intracranial arterial stenosis. N Engl J Med 365 : 993-1003, 2011

9. Derdeyn $C P$, Chimowitz MI, Lynn MJ, Fiorella D, Turan TN, Janis $L S$, et al. : Aggressive medical treatment with or without stenting in high-risk patients with intracranial artery stenosis (SAMMPRIS): the final results of a randomised trial. Lancet 383 : 333-341, 2014

10. Feigin VL, Krishnamurthi RV, Parmar P, Norrving B, Mensah GA, Bennett $D A$, et al. : Update on the global burden of ischemic and hemorrhagic stroke in 1990-2013: the GBD 2013 study. Neuroepidemiology 45 : 161-176, 2015

11. Feldmann E, Daneault N, Kwan E, Ho KJ, Pessin MS, Langenberg P, et al. : Chinese-white differences in the distribution of occlusive cerebrovascular disease. Neurology 40 : 1541-1545, 1990

12. Felling RJ, Sun LR, Maxwell EC, Goldenberg N, Bernard T : Pediatric arterial ischemic stroke: epidemiology, risk factors, and management.
Blood Cells Mol Dis 67 : 23-33, 2017

13. Ferro JM, Massaro AR, Mas JL : Aetiological diagnosis of ischaemic stroke in young adults. Lancet Neurol 9 : 1085-1096, 2010

14. GBD 2016 Causes of Death Collaborators : Global, regional, and national age-sex specific mortality for 264 causes of death, 1980-2016: a systematic analysis for the Global Burden of Disease Study 2016. Lancet 390 : 1151-1210, 2017

15. Gökben S, Tosun A, Bayram N, Serdaroglu G, Polat M, Kavakli K, et al. : Arterial ischemic stroke in childhood: risk factors and outcome in old versus new era. J Child Neurol 22 : 1204-1208, 2007

16. Grant PE, He J, Halpern EF, Wu O, Schaefer PW, Schwamm LH, et al. : Frequency and clinical context of decreased apparent diffusion coefficient reversal in the human brain. Radiology $221:$ 43-50, 2001

17. Hata J, Ninomiya T, Hirakawa Y, Nagata M, Mukai N, Gotoh S, et al. : Secular trends in cardiovascular disease and its risk factors in Japanese: half-century data from the Hisayama study (1961-2009). Circulation 128 : 1198-1205, 2013

18. Hong KS, Bang OY, Kang DW, Yu KH, Bae HJ, Lee JS, et al. : Stroke statistics in Korea: part I. epidemiology and risk factors: a report from the korean stroke society and clinical research center for stroke. J Stroke $15: 2-20,2013$

19. Imai K, Mori T, Izumoto H, Watanabe M, Majima K : Emergency carotid artery stent placement in patients with acute ischemic stroke. AJNR Am J Neuroradiol 26 : 1249-1258, 2005

20. Islam MS, Anderson CS, Hankey GJ, Hardie K, Carter K, Broadhurst R, et al. : Trends in incidence and outcome of stroke in Perth, Western Australia during 1989 to 2001: the Perth Community Stroke Study. Stroke 39 : 776-782, 2008

21. Jauch EC, Saver JL, Adams HP Jr, Bruno A, Connors JJ, Demaerschalk $B M$, et al. : Guidelines for the early management of patients with acute ischemic stroke: a guideline for healthcare professionals from the American Heart Association/American Stroke Association. Stroke 44 : 870947, 2013

22. Kainth D, Chaudhry SA, Kainth H, Suri FK, Qureshi Al : Epidemiological and clinical features of moyamoya disease in the USA. Neuroepidemiology 40 : 282-287, 2013

23. Koton S, Schneider AL, Rosamond WD, Shahar E, Sang Y, Gottesman RF, et al. : Stroke incidence and mortality trends in US communities, 1987 to 2011. JAMA 312 : 259-268, 2014

24. Krishnamurthi RV, Feigin VL, Forouzanfar MH, Mensah GA, Connor M, Bennett DA, et al. : Global and regional burden of first-ever ischaemic and haemorrhagic stroke during 1990-2010: findings from the Global Burden of Disease Study 2010. Lancet Glob Health 1 : e259-e281, 2013

25. Kuriyama S, Kusaka Y, Fujimura M, Wakai K, Tamakoshi A, Hashimoto $S$, et al. : Prevalence and clinicoepidemiological features of moyamoya disease in Japan: findings from a nationwide epidemiological survey. Stroke 39 : 42-47, 2008

26. Lackland DT, Roccella EJ, Deutsch AF, Fornage M, George MG, Howard $G$, et al. : Factors influencing the decline in stroke mortality: a statement from the American Heart Association/American Stroke Association. 
Stroke 45 : 315-353, 2014

27. Lanza G, Ricci S, Setacci C, Castelli P, Novalil C, Pratesi C, et al. : An update on Italian Stroke Organization guidelines on carotid endarterectomy and stenting. Int J Stroke 9 Suppl A100 : 14-19, 2014

28. Lee JH, Yang DH, Park HS, Cho Y, Jun JE, Park WH, et al. : Incidence of hypertension in Korea: 5-year follow-up study. J Korean Med Sci 26 : 1286-1292, 2011

29. Lee $W K$, Oh CW, Lee $H$, Lee $K S$, Park $H$ : Factors influencing the incidence and treatment of intracranial aneurysm and subarachnoid hemorrhage: time trends and socioeconomic disparities under an universal healthcare system. J Neurointerv Surg 11 : 159-165, 2019

30. Levy El, Rahman M, Khalessi AA, Beyer PT, Natarajan SK, Hartney ML, et al. : Midterm clinical and angiographic follow-up for the first Food and Drug Administration-approved prospective, single-arm trial of primary stenting for stroke: SARIS (stent-assisted recanalization for acute ischemic stroke). Neurosurgery 69 : 915-920; discussion 920, 2011

31. Levy El, Siddiqui AH, Crumlish A, Snyder KV, Hauck EF, Fiorella DJ, et al. : First Food and Drug Administration-approved prospective trial of primary intracranial stenting for acute stroke: SARIS (stent-assisted recanalization in acute ischemic stroke). Stroke 40 : 3552-3556, 2009

32. Mackay MT, Wiznitzer M, Benedict SL, Lee KJ, Deveber GA, Ganesan $V$, et al. : Arterial ischemic stroke risk factors: the international pediatric stroke study. Ann Neurol 69 : 130-140, 2011

33. Miao W, Zhao PL, Zhang YS, Liu HY, Chang Y, Ma J, et al. : Epidemiological and clinical features of Moyamoya disease in Nanjing, China.

Clin Neurol Neurosurg 112 : 199-203, 2010

34. Moon TJ : Light and shadows of the Korean healthcare system. J Korean Med Sci 27 Suppl : S3-S6, 2012

35. Murray CJ, Vos T, Lozano R, Naghavi M, Flaxman AD, Michaud C, et al. : Disability-adjusted life years (DALYS) for 291 diseases and injuries in 21 regions, 1990-2010: a systematic analysis for the Global Burden of Disease Study 2010. Lancet 380 : 2197-2223, 2012
36. Park JK, Kim KS, Kim CB, Lee TY, Lee KS, Lee DH, et al. : The accuracy of ICD codes for cerebrovascular diseases in medical insurance claims. Korean J Prev Med 33 : 76-82, 2000

37. Powers WJ, Rabinstein AA, Ackerson T, Adeoye OM, Bambakidis NC, Becker $\mathrm{K}$, et al. : 2018 guidelines for the early management of patients with acute ischemic stroke: a guideline for healthcare professionals from the American Heart Association/American Stroke Association. Stroke 49 : e46-e110, 2018

38. Roth GA, Forouzanfar MH, Moran AE, Barber R, Nguyen G, Feigin VL, et al. : Demographic and epidemiologic drivers of global cardiovascular mortality. N Engl J Med 372 : 1333-1341, 2015

39. Rothwell PM, Coull AJ, Giles MF, Howard SC, Silver LE, Bull LM, et al. : Change in stroke incidence, mortality, case-fatality, severity, and risk factors in Oxfordshire, UK from 1981 to 2004 (Oxford Vascular Study). Lancet 363 : 1925-1933, 2004

40. Sacco RL, Kargman DE, Gu Q, Zamanillo MC : Race-ethnicity and determinants of intracranial atherosclerotic cerebral infarction. The northern manhattan stroke study. Stroke 26 : 14-20, 1995

41. Setacci C, Argenteri A, Cremonesi A, de Donato G, Galzerano G, Lanza G, et al. : Guidelines on the diagnosis and treatment of extracranial carotid artery stenosis from the Italian society for vascular and endovascular surgery. J Cardiovasc Surg (Torino) 55 : 119-131, 2014

42. Sivenius J, Tuomilehto J, Immonen-Räihä P, Kaarisalo M, Sarti C, Torppa J, et al. : Continuous 15-year decrease in incidence and mortality of stroke in Finland: the FINSTROKE study. Stroke 35 : 420-425, 2004

43. Stacey A, Toolis C, Ganesan V : Rates and risk factors for arterial ischemic stroke recurrence in children. Stroke 49 : 842-847, 2018

44. Wang H, Wang D, Fraser K, Swischuk J, Elwood P : Emergent combined intracranial thrombolysis and carotid stenting in the hyperacute management of stroke patients with severe cervical carotid stenosis. AJNR Am J Neuroradiol 28 : 1162-1166, 2007 